\title{
Drive-through testing in COVID-19: experience from NHS Lothian
}

\author{
Authors: Katherine Hill, ${ }^{A}$ Robyn Campbell, ${ }^{A}$ Callum Mutch, ${ }^{A}$ Oliver Koch ${ }^{B}$ and Claire Mackintosh ${ }^{\mathrm{B}}$
}

The growing epidemic of SARS-CoV-2 challenges healthcare resources globally and mandates innovation. We describe our novel drive-through coronavirus testing which we used for testing of possible cases in the contain phase of UK response and are now using for healthcare worker testing. We found that this system was pragmatic, cost-efficient and favourable for patients. It is easily modifiable for use in future infectious disease outbreaks.

KEYWORDS: SARS-CoV-2, COVID-19, testing, health economics, innovation

DOI: $10.7861 /$ clinmed.2020-0160

\section{Background}

The growing pandemic of SARS-CoV-2 challenges healthcare resources globally. The majority of patients with COVID-19 experience a mild illness, with a minority requiring hospital admission. ${ }^{1,2}$ Thus, in the 'contain' phase of the UK response, innovation within healthcare to detect and isolate cases rapidly was required to prevent local spread and minimise unnecessary in-hospital exposure and transmission.

NHS Lothian covers a population of approximately 900,000 people. Our infectious diseases unit sits within this trust and is additionally a referral centre for high consequence infectious diseases (HCIDs) from other health boards. As the COVID-19 epidemic escalated, it became critical to preserve our specialist inpatient beds for those requiring admission and as such a protocol for community testing was rapidly developed. Here we describe our drive-through coronavirus testing for possible cases.

\section{Testing protocol}

For clinically well patients with capacity to self-isolate we devised two testing modalities: in-home testing and drive-through testing.

The drive-through testing team worked in pairs: a testing member and a support member. Once in the testing zone, the patient telephoned the control room. The testing member donned

Authors: Ainfectious diseases registrar, NHS Lothian, Edinburgh, UK; Binfectious diseases consultant, NHS Lothian, Edinburgh, UK personal protective equipment (PPE). After confirmation of patient details, a combined nose and throat swab was taken, placed into pre-labelled viral transport medium, double-bagged, and placed into a category $\mathrm{B}$ bio-bottle. The testing member returned to the doffing area with the category B container, which was cleaned. PPE was doffed into the appropriate clinical waste stream, and hands cleaned with alcohol hand-rub. The bio-bottle was placed into category B shipping box. Recommended PPE at that time

\section{Primary care}

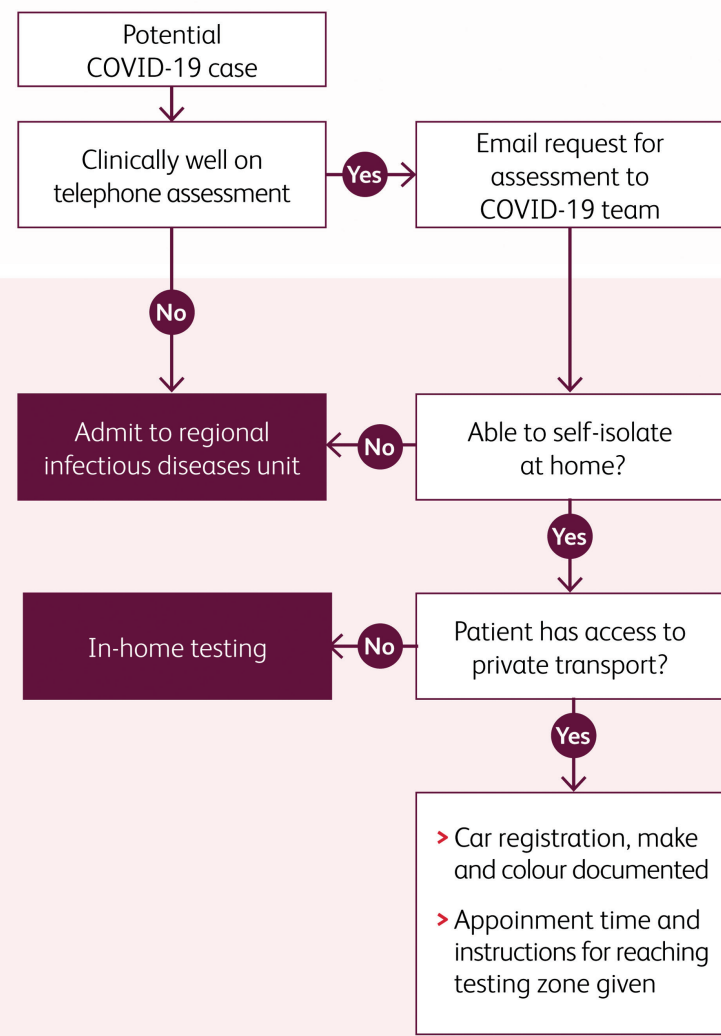

Infectious diseases and HPT

Fig 1. A possible COVID-19 case assessment flowchart. 
consisted of long-sleeved fluid-resistant gown, double-gloved nonsterile gloves, FFP3 respirator, and visor. Gown and gloves were changed between each patient, FFP3 and visor were changed at least hourly. Patients were informed of their results within 24 to 36 hours. Fig 1 shows our COVID-19 case assessment flowchart.

\section{Discussion}

This is an extremely efficient system for testing patients. Over the first 18 hours of testing, 92 patients were sampled between two staff members via the drive-through, with capacity for more. In-home testing teams were only able to test a maximum of 15 patients per 3 staff members per day. We found that, where feasible, patients preferred drive-through testing, which offers greater anonymity and specific appointment times. This pragmatic, safe and cost-efficient service facilitates quicker results, alleviating patient anxiety while also reducing pressure on inpatient NHS services. ${ }^{3}$ We have since modified this method for screening of healthcare workers.

\section{Acknowledgements}

We would like to thank the nurses and doctors that helped to set up and run the COVID-19 community testing teams.

\section{References}

1 Wu Z, McGoogan JM. Characteristics of and important lessons from the coronavirus disease 2019 (COVID-19) outbreak in China. JAMA 2020;323:1239-42.

2 Lake M. What we know so far: COVID-19 current clinical knowledge and research. Clin Med 2020;20:124-7.

3 Mark K, Steel K, Stevenson J et al. COVID-19 community testing team in Scotland: a 14-day review, 6 to 20 February 2020. Euro Surveill 2020;25:pii $=2000217$

Address for correspondence: Dr Katherine Hill, Regional Infectious Diseases Unit, NHS Lothian, Western General Hospital, Crewe Road South, Edinburgh EH4 2XU, UK. Email: k.hill@doctors.org

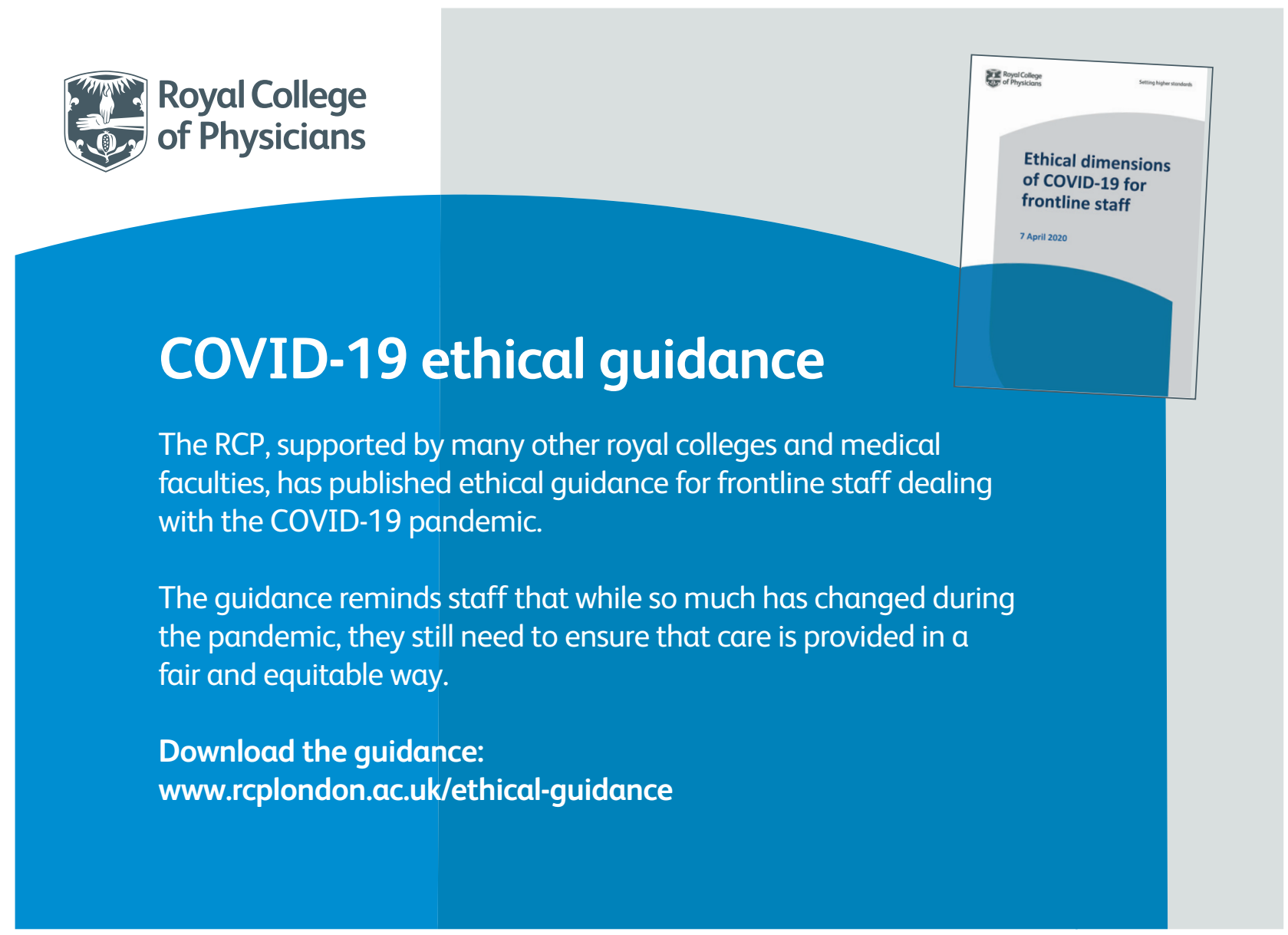




\section{Royal College of Physicians}

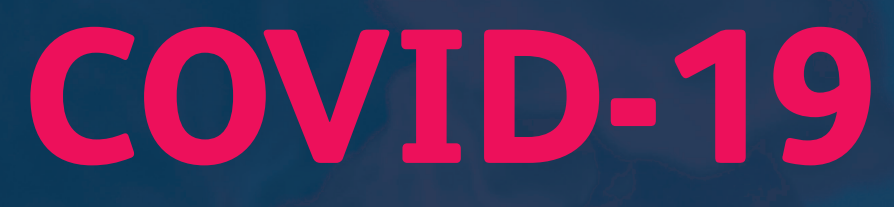

At the Royal College of Physicians we are committed to informing, supporting and advocating for our members who are at the heart of the COVID-19 pandemic and NHS response.

Our COVID-19 online hub includes information on the latest developments for clinicians and links to up-to-date guidance.

Visit the hub at 